\title{
Almost periodic and quasi-periodic solutions of differential equations
}

\section{G.C. O'Brien}

This thesis examines some problems associated with almost periodic and quasi-periodic solutions of differential equations. The first half is concerned with qualitative aspects while the second half is devoted to socalled "small divisor" problems.

The first chapter of interest examines almost periodic solutions of the almost periodic differential equation

$$
x^{\prime}=\psi(x, t)
$$

where $x \in R^{p}$ and $\psi(x, t)$ is almost periodic in $t$, uniformly for $x$ in any bounded subset of $R^{p}$. It is shown that if $\phi(t)$ is any almost periodic solution of ( 1 ) then the codimension of the frequency basis of $\psi(x, t)$ with respect to the frequency basis of $\phi(t)$ is less than $p$. The proof is simpler and more direct than previous similar results and does not use topological dynamics or the concept of translation numbers. Furthermore, the proof constructs a whole family of almost periodic solutions related to the given solution.

The next chapter concerns a result due to Cherry. Cherry considered holomorphic autonomous differential equations with an integral invariant in p-dimensional euclidean space. He purported to show that all solutions of such equations in a $p$-dimensional compact invariant set were quasiperiodic. We provide partial counter examples to Cherry's assertions and show under more general conditions than Cherry that quasi-periodic solutions of a real hamiltonian system have at most $\frac{p}{2}$ basic frequencies.

Received $17 \mathrm{July}$ 1972. Thesis submitted to the Australian National University, March 1972. Degree approved, July 1972. Supervisor: Mr W.A. Coppel. 
In the next chapter we construct an iterative procedure for finding a change of variables to reduce the linear system

$$
\begin{aligned}
& x^{\prime}=A x+P(\phi) x, \\
& \phi^{\prime}=\omega,
\end{aligned}
$$

where $x \in R^{n}, \omega \in R^{m}$ and $P(\phi)$ is holomorphic, to a system with constant coefficients. Under various assumptions on the eigenvalues of $A$ and their relationship with $\omega$ we use the technique of accelerated convergence to overcome the difficulty of small divisors and show that the iteration converges and that the change of variables is itself quasiperiodic.

The final chapter is an extension of the results of the previous chapter to the case when $P(\phi)$ is $Z$ times differentiable. We approximate the differentiable functions by holomorphic functions and by carefully selecting the exponent for the accelerated convergence prove that an iterative change of variables again converges to a quasi-periodic transformation which reduces the initial system to one with constant coefficients. As is always the case in such problems, there is an inevitable loss of derivatives. The best previous result, which is due to Mitropol'skī̌ and Samoǐlenko, required

$$
Z>\frac{k}{(k-1)(2-k)}[\kappa(m+\tau)+2 m+2],
$$

where $k$ is the exponent of accelerated convergence $(1<\kappa<2)$ and $\tau$ is a constant occurring in the relationship between the eigenvalues of $A$ and $\omega$. Our result requires only that

$$
\tau>\tau \text {. }
$$

